\title{
Shape Determination for Deformed Cavities
}

\author{
Lie-Quan Lee*, Volkan Akcelik*, Sheng Chen*, Lixin Ge*, Zenghai Li*, Cho Ng*, Liling Xiao*, \\ Kwok Ko*, Omar Ghattas**
}

\begin{abstract}
A realistic superconducting RF cavity has its shape deformed comparing to its designed shape due to the loose tolerance in the fabrication process and the frequency tuning for its accelerating mode. A PDE-constrained optimization problem is proposed to determine the deformation of the cavity. A reduce space method is used to solve the PDE-constrained optimization problem where design sensitivities were computed using a continuous adjoint approach. A proof-of-concept example is given in which the deformation parameters of a single cavity-cell with two different types of deformation were computed.
\end{abstract}

Index Terms-Deformation, Inverse problem, Superconducting RF cavities.

\section{INTRODUCTION}

$\mathrm{T}$ HE International Linear Collider is a proposed new electron-position collider with efforts of hundreds of accelerator scientists and particle physicists in North America, Europe and Asia [10]. The nature of the ILC's electronpositron collisions would give it the capability to answer compelling questions such as the identity of dark matter and the existence of extra dimensions. The heart of the ILC is the superconducting Radio Frequency (RF) Linac which is about $30 \%$ of the total cost of the ILC. The Linac consists of 20,000 superconducting RF cavities. Due to prohibitively high cost, the production tolerance in fabricating cavities is very high. The tuning procedure which makes the accelerating frequency correct also changes the shape of the cavity. For example, as described in [11] for making TESLA cavities, the production starts from niobium sheets to make half cells without accurately testing and correcting mechanical length. One assembled dumb-bells from two half cells by welding them at the iris together with stiffening ring and trim the dumb-bells at the equator to adjust the accelerating frequency. The next step is assembling eight dumb-bells with end cups and beam pipes to make a complete a 9-cell TESLA cavity. In the final step, one tunes different parts of the 9-cell cavity to get the correct resonance frequency and flat field of the accelerating mode.

Both production procedure and tuning procedure change the shapes of the designed cavity which leads to significantly

This work was supported in part by the U.S. Department of Energy under contract DE-AC02-76SF00515.

* Advanced Computations Department, Stanford Linear Accelerator Center, Menlo Park, CA 94025

** Institute for Computational Engineering and Sciences, the University of Texas at Austin, Texas 78712 change in HOM frequency and field distribution and the HOM damping effect. Therefore, it is of extreme importance to know the true dimensions of the realistic cavities in order for reliable prediction of the HOM damping.

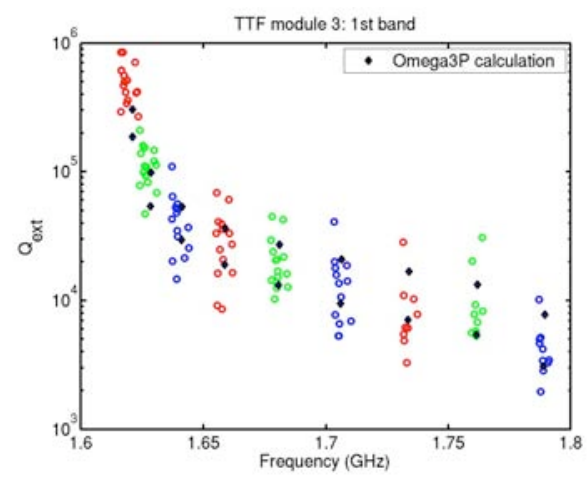

Figure 1. The measured frequencies and external Q's from TTF module 3 [12] versus the computed counterparts from the ideal cavity.

\section{METHODS}

\section{A. PDE-Constrained Optimization}

Let $\mathrm{n}$ denote the number of eigen-frequencies $(f)$ and $\mathrm{m}$ denote the number of independent deformation parameters (d), the shape determination problem is to minimize the following objective $F$ :

$F=\sum_{i=1}^{n} \alpha_{i}\left(f_{i}\left(d_{1}, \ldots, d_{m}\right)-f_{i}^{*}\right)^{2}+\sum_{i=1}^{m} \sum_{j=1}^{p} \beta_{i j}\left(\left|\overrightarrow{\mathbf{E}}^{j}\left(r_{i}\right)-\overrightarrow{\mathbf{E}}_{i}^{j}\right|\right)$

subject to the PDE constraints in the domain $\Omega(\mathbf{d})$, electric boundary $\boldsymbol{\Gamma}_{E}$ and magnetic boundary $\boldsymbol{\Gamma}_{M}$ :

$$
\left\{\begin{aligned}
\boldsymbol{\nabla} \times\left(\frac{1}{\epsilon} \boldsymbol{\nabla} \times \mathbf{H}_{i}\right)-\lambda_{i} \mu \mathbf{H}_{i} & =\mathbf{0} \text { in } \Omega(\mathbf{d}), \\
\boldsymbol{\nabla} \cdot \mathbf{H}_{i} & =0 \text { in } \Omega(\mathbf{d}), \\
\mathbf{n} \times \mathbf{H}_{i} & =\mathbf{0} \text { on } \Gamma_{M}(\mathbf{d}), \\
\mathbf{n} \times\left(\frac{1}{\epsilon} \nabla \times \mathbf{H}_{i}\right) & =\mathbf{0} \text { on } \Gamma_{E}(\mathbf{d}), \\
\frac{1}{2} \int_{\Omega(\mathbf{d})} \mu \mathbf{H}_{i} \cdot \mathbf{H}_{i} d \Omega(\mathbf{d})-\frac{1}{2} & =0
\end{aligned}\right.
$$

where $f_{i}$ is the computed eigen-frequency of the $i$ th mode and $f_{i}^{*}$ is the corresponding measured eigen-frequency. $\boldsymbol{H}_{i}$ is the magnetic field for the $i$ th eigen-mode. $\varepsilon$ is the permittivity and

Lie-Quan Lee is the corresponding author at the Stanford Linear Accelerator Center, Menlo Park, CA 94025 USA. Phone: 650-926-5356; fax: 650-926-4603; e-mail: liequan@ slac.stanfrod.edu 
$\mu$ is the permeability. Note that eigenvalue $\lambda_{i}=\left(\frac{2 \pi f_{i}}{c}\right)^{2}$ where $c$ is the speed of light.

To solve the above PDE constrained optimization problem, we use reduced space method [1]. We compute the design sensitivities using a continuous adjoint approach [2][3]. Given the cavity shape, we solve the forward problem (i.e., the above PDE problem) [2] and adjoint problems. We compute the reduced gradient through integral equations evaluated at the boundary of the cavity. All of these have been implemented in our parallel finite element based code Omega3P []. We will discuss in details how we solve the forward problem, the adjoint problem and how we evaluate the reduced gradient.

\section{B. The Forward Problem}

There have been extensive studies on how to numerically solve the harmonic Maxwell's equations (2). The use of Nedelec edge elements [] guarantees that solutions of the discretized problem from (2) with nonzero $\lambda$ are divergencefree and physical [4][5]. In our simulations, we used a set of higher-order Nedelec type elements [6] to discretize (2) in Omega3P. If we expand the magnetic field $\mathbf{H}$ with the basis function set $\left\{\overrightarrow{\mathbf{N}}_{i}\right\}$ as follows:

$$
\mathbf{H}=\sum_{i=1}^{n} c_{i} \overrightarrow{\mathbf{N}}_{i}
$$

We will get the following eigenvalue problem from the finite element procedure:

$$
\begin{aligned}
& \mathbf{K} c=\lambda \mathbf{M} c \\
& \mathbf{K}_{i j}=\int_{\Omega}\left(\nabla \times \overrightarrow{\mathbf{N}}_{i}\right) \cdot \frac{1}{\epsilon}\left(\nabla \times \overrightarrow{\mathbf{N}}_{j}\right) d \Omega \\
& \mathbf{M}_{i j}=\int_{\Omega} \mu \overrightarrow{\mathbf{N}}_{j} \cdot \overrightarrow{\mathbf{N}}_{j} d \Omega
\end{aligned}
$$

Note that matrix $\mathbf{K}$ and $\mathbf{M}$ are symmetric while $\mathbf{M}$ is also positive definite. In the end-to-end accelerator cavity system simulations, these matrices can each be of size corresponding to 100's million of degrees of freedom. An parallel shift-andinvert Lanczos method is implemented in Omega3P to solve (4) in the parallel computers [8][9].

\section{The Adjoint Problem}

Let us consider only one frequency and the Lagrangian functional is

$$
\begin{aligned}
& \mathcal{L}\left(\mathbf{H}, \lambda, \mathrm{d}, \mathbf{T}, \wedge_{M}, \wedge_{E}, \xi\right)=\left(f-f^{*}\right)^{2} \\
& +\int_{\Omega} \mathbf{T} \cdot\left(\nabla \times \frac{1}{\epsilon} \nabla \times \mathbf{H}-\lambda \mu \mathbf{H}\right) d \Omega \\
& +\int_{\Gamma_{M}} \wedge_{M} \cdot(n \times \mathbf{H}) d \Gamma \\
& +\int_{\Gamma_{E}} \wedge_{E} \cdot\left(n \times \frac{1}{\epsilon} \nabla \times \mathbf{H}\right) d \Gamma \\
& +\frac{\xi}{2}\left(\int_{\Omega} \mathbf{H} \cdot \mathbf{H} d \Omega-1\right)
\end{aligned}
$$

where $\mathbf{T}, \xi, \Lambda_{\mathrm{M}}$ and $\Lambda_{\mathrm{E}}$ are the adjoint variables. To obtain the adjoint system, we take the variation of the Lagrangian with respect to state variable $\mathbf{H}$ and require that the result vanish. We will get the following adjoint problem:

$$
\begin{aligned}
& \nabla \times\left(\frac{1}{\epsilon} \nabla \times \mathbf{T}\right)-\lambda \mu \mathbf{T}+\mu \xi \mathbf{H}=0 \text { in } \Omega \\
& n \times \mathbf{T}=0 \text { on } \Gamma_{M} \\
& n \times \frac{1}{\epsilon} \nabla \times \mathbf{T}=0 \text { on } \Gamma_{E} \\
& \int_{\Omega} \mathbf{T} \cdot \mathbf{H} d \Omega=\frac{d}{d \lambda}\left(f-f^{*}\right)^{2}
\end{aligned}
$$

We use the same set of the basis function set $\left\{\overrightarrow{\mathbf{N}}_{i}\right\}$ to expand the adjoint variable $\mathrm{T}$ :

$$
\mathbf{T}=\sum_{i=1}^{n} t_{i} \vec{N}_{i}
$$

And we get the following algebraic equation:

$$
\begin{aligned}
& (\mathbf{K}-\lambda \mathbf{M}) \mathbf{t}+\xi \mathbf{M c}=0 \\
& (\mathbf{M c})^{t} \mathbf{t}=\frac{d}{d \lambda}\left(f-f^{*}\right)^{2}
\end{aligned}
$$

where $(\lambda, \mathbf{c})$ is the eigenpair. By multiplying $\mathbf{c}^{\mathrm{t}}$ on the both size of the first equation and comparing to the state eigenvalue problem in (4), it immediately gives us that $\xi$ is equal to zero. Along with the second equation, we get that

$$
\mathbf{t}=\mathbf{c} \frac{d}{d \lambda}\left(f-f^{*}\right)^{2}
$$

Note that we did not explicitly enforce the divergence-free condition of $\mathbf{H}$ field in the Lagrangian. This is because we use the Nedelec elements which guarantee that the corresponding $\mathbf{H}$ is divergence free for a nonzero eigenvalue. This is also true for the adjoint variable $\mathbf{T}$. This point can be seen by taking divergence of the first equation in (5).

For the case that there are multiple frequencies involved in the objective function (1), we will multiple adjoint systems with each adjoint system corresponding to one eigenpair.

\section{Reduced Gradient Computation}

According to [3], we can write out the reduce gradient to be the following:

$$
\begin{aligned}
& \frac{\partial \mathcal{L}}{\partial d_{i}}=\int_{\Gamma}\left(\mathbf{V}_{i} \cdot n\right)\left[\frac{1}{\epsilon}(\nabla \times \mathbf{H}) \cdot(\nabla \times \mathbf{T})-\lambda \mu \mathbf{T} \cdot \mathbf{H}+\frac{1}{2} \xi \mu \mathbf{H} \cdot \mathbf{H}\right] d \Gamma \\
& +\int_{\Gamma_{M}}\left(\mathbf{V}_{i} \cdot n\right)(n \cdot \nabla+\nabla \cdot n)\left[\mathbf{T} \cdot\left(n \times \frac{1}{\epsilon} \nabla \times \mathbf{H}\right)+\mathbf{H} \cdot\left(n \times \times \frac{1}{\epsilon} \nabla \times \mathbf{T}\right)\right] d \Gamma
\end{aligned}
$$

where $\mathbf{V}_{i}$ is the so-called design velocity, namely, the derivative of the boundary coordinate with respect to a deformation parameter. $\nabla \cdot \mathrm{n}$ is the mean curvature and the normal derivative $n \cdot \nabla$ operates on the term in square brackets. In the simulations, the second term in (10) often vanish either because there is no symmetry in the simulated cavity or because $\left(\mathbf{V}_{i} \cdot \mathrm{n}\right)$ is zero.

\section{E. Parallel Implementation}

In determinating the deformed shape of an ideal cavity, we first create the CAD model of the ideal cavity. From the CAD model, we generate tetrahedral mesh in the domain using mesh generation tool cubit [13] and save the mesh in NetCDF [14] format. We used CFSQP [15] as the optimization engine which only requires a function to evaluate the objective function and a function to compute the gradient. In order to evaluate the objective function (1), we solve the forward eigenvalue problem in parallel due to large problem size. In computing the gradients, we get the adjoint variables using (9) and evaluate the gradient (10) using Gaussian ineetrals. We 
replicate the optimization engine is in each MPI process due to a relatively small number of deformation parameters. In evaluating (10), we compute design velocity analytically. Once we get a new set of deformation parameters, we move the mesh analytically for the next step of the optimization.

\section{EXPERIMENTS}

In this experiment, we has a single cell from TDR cavity of International Linear Collider[] as shown in Figure 2. We consider two types of deformations. One is so-called XYdeformation to make the xy cut-plane of the cell from circular shape to an elliptical shape. It controlling parameter is denoted as $\mathrm{d}_{1}$. The other type is Z-deformation and it introduce different cell length for the left cup of the cell. Its controlling parameter is denoted as $d_{2}$. Figure 3 and 4 illustrates the two types of the deformation, respectively.

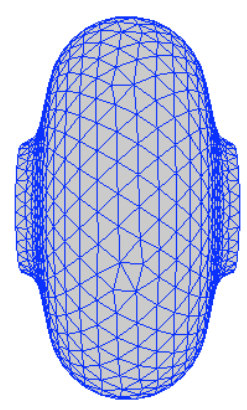

Figure 2. The ideal shape of a single cell from TDR cavity and its mesh.

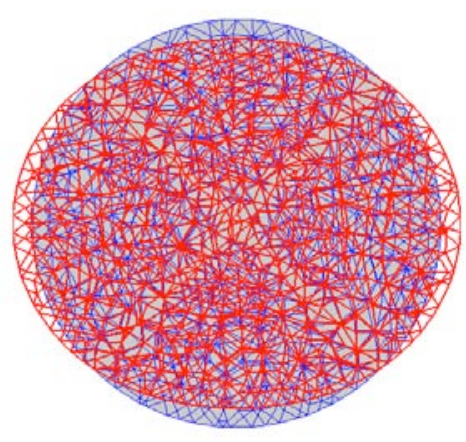

Figure 3. The elliptical shape of the xy cut-plane of a deformed cell.

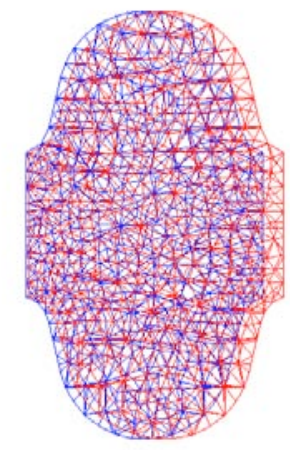

Figure 4. The z-cut plane of the deformed cell.

In this example, we used $23 \mathrm{~K}$ tetrahedral mesh and linear element and wanted the first pair of the dipole modes to be $1.823896082168882 \mathrm{GHz}$ and $1.824157787219089 \mathrm{GHz}$. Note that the above frequencies correspond to the first dipole pair of the deformed cell with the both XY-deformation parameter and Z-deformation parameter being 100 micron. We assigned the initial guess of $d_{1}$ and $d_{2}$ to be 10 micron. The two dipole modes have frequency of $1.824730649724285 \mathrm{GHz}$ and $1.824863516314319 \mathrm{GHz}$, respectively. After 13 optimization iterations, we got that the frequencies of the dipole modes being $1.823896071983175 \mathrm{GHz}$ and 1.824157795319187 $\mathrm{GHz}$, respectively while $\mathrm{d}_{1}$ is 100.005 micron and $\mathrm{d}_{2}$ is 100.000 micron. Note that the frequencies at the optimal point are very close to the targeted values.

This proof-of-concept example gives us the confidence that we have a correct approach to tackle the shape uncertainty in ILC cavity and further study of the whole ILC cavity with different patterns of deformations will lead to great insight to actual beam kick due to HOM in the operation.

\section{SUMMARY}

A realistic superconducting $\mathrm{RF}$ cavity has its shape deformed comparing to its designed shape due to the loose tolerance in the fabrication process and the frequency tuning for its accelerating mode. A PDE-constrained optimization problem is proposed to determine the deformation of the cavity. A reduce space method is used to solve the PDEconstrained optimization problem where design sensitivities were computed using a continuous adjoint approach. A proofof-concept example is demonstrated in which the deformation parameters of a single cavity-cell with two different types of deformation were computed.

\section{ACKNOWLEDGMENT}

This research used resources of the National Center for Computational Sciences at Oak Ridge National Laboratory, which is supported by the Office of Science of the U.S. Department of Energy under Contract No. DE-AC0500OR22725. This research also used resources of the National Energy Research Scientific Computing Center, which is supported by the Office of Science of the U.S. Department of Energy under Contract No. DE-AC03-76SF00098. 


\section{REFERENCES}

[1] V. Akcelik, G. Biros, O. Ghattas, J. Hill, D. Keyes, and B. van Bloemn Waanders, "Parallel algorithms for PDE-constrained optimization", in Frontiers of Parallel Computing, M. Heroux, P. Raghaven, and H. Simon, eds, SIAM, 2006, to be published.

[2] V. Akcelik, G. Biros, O Ghattas, D. Keyes, K. Ko, L. Lee and E. G. $\mathrm{Ng}$, "Adjoint methods for electromagnetic shape optimization of the low-loss cavity for the International Linear Collider", Journal of Physics: Conference Series 16, pp435-445, 2005

[3] O. Pironneau, "Optimal shape design for elliptic systems", SpringerVerlag, 1983.

[4] J. Jin, "The finite element method in Electroganetics", second edition, John Wiley \& Sons, INC, New York, 2002

[5] P. Monk, "Finite Element Methods for Maxwell's Equations", Oxford, 2003

[6] D.-K. Sun, J.-F. Lee, and Z. Cendes, "Construction of nearly orthogonal nedelec bases for rapid convergence with multilevel preconditioned solvers," SIAM Journal on Scientific Computing, vol. 23 , no. 4, pp. 1053-1076, 2001

[7] J. C. Nedelec, "Mixed finite elements in R3", Numer. Meth., Vol. 35, pp. 315-341, 1980

[8] L. Lee, L. Ge, M. Kowalski, Z. Li, C. Ng, G. Schussman, M. Wolf, and K. Ko, "Solving large sparse linear systems in end-to-end accelerator structure simulations", in Proc. of the $18^{\text {th }}$ International Parallel and Distributed Processing Symposium, Santa Fe, New Mexico, 2004

[9] Y. Sun, N. Folwell, Z. Li, and G. Golub, "High precision accelerator cavity design using the parallel eigensolver Omega3P," in Proc. of the $18^{\text {th }}$ Annual Review of Progress in Applied Computational Electromagnetics, 2002.

[10] The Interational Linear Collider webpage. http://www.linearcollider.org

[11] G. Kreps, D. Proch, J. Sekutowicz, "Half-cell and dumb-bell frequency testing for the correction of the TESLA cavity length", in Procs. Of the $9^{\text {th }}$ Workshop on RF Superconductivity, pp. 400, Santa Fe, New Mexico, USA 1999

[12] J. Sekutowicz, ILC Workshop, KEK, Japan, Nov. 13-15, 2004

[13] CUBIT geometry and mesh generation toolkit, http://cubit.sandia.gov

[14] The network Common Data Form (NetCDF), http://www.unidata.ucar.edu/software/netcdf/

[15] C. T. Lawrence, J. L. and A. L. Tits, "CFSQP software", 1998 